\title{
Consultations in Molecular Diagnostics
}

\section{Influence of the Duplication of CFTR Exon 9 and Its Flanking Sequences on Diagnosis of Cystic Fibrosis Mutations}

\author{
Ayman El-Seedy, ${ }^{*}$ Tony Dudognon, ${ }^{*}$ \\ Frédéric Bilan, ${ }^{*}$ Marie-Claude Pasquet, ${ }^{\dagger}$ \\ Marie-Pierre Reboul, ${ }^{\ddagger}$ Albert Iron, ${ }^{\ddagger}$ Alain Kitzis, ${ }^{*}$ \\ and Véronique Ladeveze* \\ From the Institut de Physiologie et Biologie Cellulaires," Centre \\ National de la Recherche Scientifique Unité Mixte de Recherche \\ 6187, Université de Poitiers, Poitiers; the Centre Hospitalia \\ Universitaire de Poitiers, ${ }^{\dagger}$ Poitiers; and the Laboratoire de \\ Génétique Moléculaire, ${ }^{\ddagger}$ CHU de Bordeaux, Bordeaux, France
}

The DNA sequences of seven regions in the human genome were examined for sequence identity with exon 9 of the cystic fibrosis transmembrane conductance regulator (CFTR) gene, which is mutated in cystic fibrosis, and its intronic boundaries. These sequences were $95 \%$ to $96 \%$ homologous. Based on this nucleotide sequence similarity, PCR primers for CFTR exon 9 can potentially anneal with other homologous sequences in the human genome. Sequence alignment analysis of the $C F T R$ exon 9 homologous sequences revealed that five registered mutations in the Cystic Fibrosis Mutation Database may be due to the undesired annealing of primers to a homologous sequence, resulting in inappropriate PCR amplification. For this reason, we propose that certain pseudomutations may result from the similarity between CFTR exon 9 (and its flanking introns) and related sequences in the human genome. Here we show that two mutations previously described in the CFTR database $($ c.1392 + 6insC; c.1392 + $12 \mathrm{G}>\mathrm{A})$ were inappropriately attributed to two individuals who sought carrier testing. A more detailed study by either direct sequencing or subcloning and sequencing of PCR products using specially designed primers revealed that these apparent mutations were not, in fact, present in CFTR. In addition, we present new PCR conditions that permit specific amplification of CFTR exon 9 and its flanking regions. (J Mol Diagn 2009, 11:488-493; DOI: 10.2353/jmoldx.2009.090005)
The cystic fibrosis (CF) gene, encoding the cystic fibrosis transmembrane conductance regulator (CFTR), is located on the long arm of chromosome 7, at position 7p31. CFTR is involved in the active transport of ions through the apical membrane of epithelial cells. ${ }^{1}$ The $250-\mathrm{kb}$ gene, containing 27 exons, appears highly susceptible to mutations due to its large size. ${ }^{2}$ More than 1500 genetic alterations have been described to date. Most are disease-causing mutations; about half lead to amino acid substitutions (missense mutations), 20\% lead to splicing errors, and 30\% appear to be nonsense and frame shift (including small deletions and insertions) or promoter mutations (http://www.genet.sickkids.on.ca/cftr/, last accessed November 29, 2006). Moreover, the type and distribution of mutations vary substantially between populations. ${ }^{3,4}$

Previous studies on the CFTR gene have reported that exon 9 and its flanking introns are present in multiple copies in the human genome. Indeed, this region is part of the large duplicated sequence unit LCR7-20 (low-copy repeats 7 to 20), which is dispersed on different chromosomes in human genome..$^{5,6}$

Screening for CFTR exon 9 mutations is difficult due to the polymorphism of the (TG)m (T)n repeats located at the end of intron 8. This variation eludes common PCRbased techniques for mutation detection in this region, including direct sequencing, as well as denaturing highperformance liquid chromatography. Thus, if denaturing high-performance liquid chromatography analysis is used, it is necessary to use primers that have been documented $^{7}$ to prevent the variability that T/TG repeats can cause. Using this method, however, only the beginning of exon 9 is amplified. Because this region of CFTR has been duplicated in several regions of the genome, we suggest that using these classical primers could lead to misidentification of a CFTR pseudogene mutation as a CF-causative mutation.

\footnotetext{
Supported by Poitiers Hospital and University of Poitiers, France.
}

Accepted for publication April 21, 2009.

Address reprint requests to Alain Kitzis, CHU de Poitiers, BP 577 , 86021 Poitiers, France. E-mail: a.kitzis@chu-poitiers.fr. 
Here, we analyzed two cases in which there were molecular diagnosis difficulties in the exon 9 region of CFTR. We demonstrate that technical anomalies leading to incorrect molecular diagnosis are due to the primers used in the PCR protocol. The consequence of these primer mismatches could be that multiple mutations already registered in the Cystic Fibrosis Mutation Database (http://www.genet.sickkids.on.ca/cftr/; last accessed December 24, 2008; Hospital for Sick Children, Toronto, Canada) could be irrelevant.

To our knowledge, there has been no previous report demonstrating the effect of sequence similarity between CFTR exon 9 (encoding a part of the first nucleotidebinding domain ${ }^{8}$ ) and related sequences on CFTR mutation screening. Therefore, our primary aim in this paper is to provide evidence of mutations in the database that are in fact pseudomutations in duplicated regions of the genome, with normal sequences in exon 9 of the CFTR gene. Since traditional methods for amplification of exon 9 and its flanking sequences will amplify several ectopic regions on chromosomes other than chromosome 7 , we define here conditions that can be used to study this region exclusively. We suggest that each patient who presents such mutations should be re-examined by our proposed method.

\section{Cases}

The first case was a pregnant woman (UNP 10186, French origin) tested at Poitiers Hospital, because her husband was heterozygous for a mutation in CFTR. The c.1392 + 6insC and c.1392 + 12G >A mutations were identified in intron 9 (T. Bienvenu, C. Cazaneuve, J. C. Kaplan, B. Neldjord, personal communication). Following this result, the parents of case 1 were tested at Bordeaux Hospital. Surprisingly, only one mutation was identified in her father (p.Asp443Tyr in exon 9), and no mutation was found in her mother. The same result (p.Asp443Tyr mutation) was obtained in Bordeaux with case 1. Using microsatellites analysis, the Bordeaux laboratory confirmed that no error of sampling (blood or DNA) was made between case 1 and her father in Poitiers or Bordeaux. For these reasons, Poitiers Hospital analyzed this case again using the original blood sample and a freshly drawn blood sample. The new analysis for this family confirmed the previous results: case 1 carries the c.1392 + 6insC and c. $1392+12 \mathrm{G}>\mathrm{A}$ mutations in intron 9, but not the Asp443Tyr mutation in exon 9. On analysis of fresh parental samples at Bordeaux Hospital, the previous parental results were also confirmed, showing that her father must be carrier for Asp443Tyr mutation in exon 9.

The second case was a pregnant woman (UNP 10481, French origin) tested in Poitiers Hospital for genetic counseling of cystic fibrosis. Her family history revealed that her cousin had died of this disease, but the underlying CFTR mutations had not been identified. The presence of mutations in the 27 exons and neighboring intronic regions of the CFTR gene was assessed by denaturing gradient gel electrophoresis (for exons $3,4,6 b, 10,11$, $12,14 \mathrm{a}, 20,21)$ and by denaturing high-performance liquid chromatography (all others). The c.1392 + 6insC and c.1392 + 12G>A mutations were detected by sequencing. Moreover, no other mutations were identified.

Control groups used in these studies consist of: (1) six individuals with no history of CF, as a negative control, and (2) 30 patients with at least one clearly identified pathogenic CFTR mutation, as a positive control. The control samples were screened using the same methods used to detect the c.1392 + 6insC and c. $1392+12 \mathrm{G}>\mathrm{A}$ mutations in cases 1 and 2 .

\section{Materials and Methods}

\section{DNA Extraction and Sequencing}

Blood samples $(5 \mathrm{ml})$ were collected in tubes containing EDTA. All genomic DNA samples were extracted from peripheral blood cells using the QIAamp DNA Blood Mini kit (Qiagen) according to the supplier's protocol. The concentration of DNA in the samples was determined by spectrophotometry to obtain the desired final concentration (5 mg/L for patients and controls [calibrator], and 10 $\mathrm{mg} / \mathrm{L}$ for control samples used for standard curve). DNA samples were screened for mutations within the CFTR gene by direct sequencing using the PCR primers as described in Table 1. PCR products were purified on Dye Ex 2.0 Spin kit columns (Qiagen) and then subjected to automated sequence analysis, on an ABI Prism 310 Genetic Analyzer using the ABI PRISM Big Dye Terminator Cycle Sequencing Kit (PE Applied Biosystems) with targeted primers.

\section{Primer Design}

CFTR gene exon 9 primers were tested for their ability to amplify CFTR exon 9 sequences from genomic DNA without interference from other sequences in the human genome. A map of primers in intron 8, exon 9, and intron 9 is shown in Figure 1. Primer sequences, annealing temperatures, and sizes and localization of the PCR products are shown in Table 1.

\section{PCR Amplification}

Genomic DNA used for mutation screening was amplified in a total volume of $50 \mu \mathrm{l}$ containing $100 \mathrm{ng}$ DNA, 1.5 $\mathrm{mmol} / \mathrm{L} \mathrm{MgCl}_{2}, 200 \mathrm{mmol} / \mathrm{L}$ dNTP, $1 \cup$ Taq polymerase (Amersham), and $20 \mathrm{pmol}$ of each primer. The conditions used for amplification were as follows: 5 minutes at $94^{\circ} \mathrm{C}$; 30 cycles of 30 seconds at $94^{\circ} \mathrm{C}$ followed by 30 seconds at annealing temperature (see Table 1), and 30 seconds at $72^{\circ} \mathrm{C}$; and a final extension step for 5 minutes at $72^{\circ} \mathrm{C}$.

\section{Subcloning and DNA Sequencing}

PCR products were subcloned into PGEM-T vector (Promega) to provide templates. Plasmid DNA was extracted by alkaline lysis using a plasmid Mega purification kit 
Table 1. Primers Used in PCR Amplification and Sequencing of CFTR Exon 9 and its Intronic Boundaries

\begin{tabular}{|c|c|c|c|c|}
\hline $\begin{array}{l}\text { PCR } \\
\text { Product }\end{array}$ & Primers names & $\begin{array}{l}\text { Primer sequence } \\
\text { (forward primer first) }\end{array}$ & $\begin{array}{l}\text { Amplicon } \\
\text { size (bp) }\end{array}$ & $\begin{array}{l}\text { Annealing } \\
\text { temp. }\left({ }^{\circ} \mathrm{C}\right)\end{array}$ \\
\hline$A$ & $\begin{array}{l}\text { CF9.6 } \\
\text { CF9 }+58\end{array}$ & $\begin{array}{l}\text { 5'-TGGGGAATTATTTGAGAAAGC-3' } \\
5^{\prime}-\text {-CCTTCCAGCACTACAAACTAGAAA - } 3^{\prime}\end{array}$ & 285 & 50 \\
\hline B & $\begin{array}{l}\text { CF9-130 } \\
\text { CF9 }+58\end{array}$ & $\begin{array}{l}\text { 5'-ACAGTGTAATGGATCATGGGC-3' } \\
\text { 5'-CCTTCCAGCACTACAAACTAGAAA-3' }\end{array}$ & 395 & 55 \\
\hline $\mathrm{C}^{\star \dagger}$ & $\begin{array}{l}\text { CF9-268 } \\
\text { CF9 }+121\end{array}$ & $\begin{array}{l}5^{\prime}-\text { TGTATACATGTAGTAATTCAGT-3' } \\
5^{\prime}-\text { ACATTCTCCTAATGCTCATG-3' }\end{array}$ & 572 & 55 \\
\hline$D^{* \dagger}$ & $\begin{array}{l}\text { CF9-70 } \\
\text { CF9 }+121\end{array}$ & $\begin{array}{l}\text { 5'-GTACATAAAACAAGCATCTAT-3' } \\
\text { 5'-TGTATACATGTAGTAATTCAGT-3' }\end{array}$ & 394 & 56 \\
\hline$E^{\star \ddagger}$ & $\begin{array}{l}\text { CF9-70 } \\
\text { CF9 }+437\end{array}$ & $\begin{array}{l}\text { 5'-GTACATAAAACAAGCATCTAT-3' } \\
5^{\prime}-\text { TCTAAATCTATTGAAAATTG-3' }\end{array}$ & 710 & 54 \\
\hline $\mathrm{F}$ & $\begin{array}{l}\text { CF9-184 } \\
\text { CF9.181 }\end{array}$ & $\begin{array}{l}5^{\prime}-\text { CCTCTAGAAACCGTATGC-3' } \\
5^{\prime}-\text { TGCCTGCTCCAGTGGAT-3' }\end{array}$ & 364 & 54 \\
\hline
\end{tabular}

*Indicates specific PCR amplification for CFTR exon 9 and its flanking introns.

†Two sets of primers were used in which the forward primers are outside the region of similarity, but the reverse primers are inside this region.

${ }^{\ddagger}$ Another set of primers was used in which both the forward and reverse primers are outside the region of similarity. This primer design allows the selective amplification of the target region, avoiding amplification of any similar sequences.

(Qiagen). The inserted DNA was subjected to automated DNA sequencing (as described above). Sequences were deduced from data obtained for both strands.

\section{Nucleotide Sequence Analysis}

Sequence homology searches against CFTR exon 9 and flanking intronic regions were executed by means of BLAST (Basic Local Alignment Search Tool) computer-based analyses, made available through the National Center for Biotechnology Information. ${ }^{9,10}$ Surprisingly, these sequences had high identity with sequences on other chromosomes. Another similarity was observed in the intronic boundaries of CFTR exon 9. These sequences were aligned/compared with human sequences in the GenBank database using the CLUSTALW program (www.ebi.ac.uk/Tools/clusta/w2/index. html, last accessed 24/12/2008), which inserts gaps into one or the other sequence in a pair wise comparison with maximize similarities between the two sequences.

\section{Data Bank Accession Numbers}

Sequences homologous to CFTR exon 9 and its flanking introns analyzed in this study were obtained from GenBank accession numbers: NW_001839181.1 Hs9_

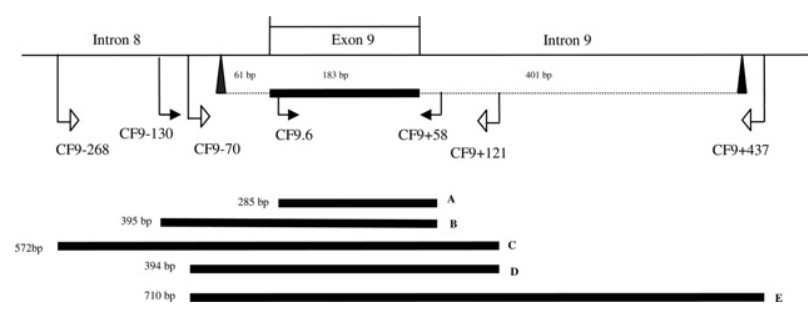

Figure 1. Schematic illustration of primers spanning exon 9 of the human CFTR gene and its intronic boundaries. Black bars, CFTR exon 9 coding region; dotted bars, intronic boundary region; solid arrowheads, beginning and end of similarity with other sequences in the human genome. DNA fragments were obtained using primers from the laboratories of Poitiers Hospital (A) and Bordeaux Hospital (B). Open arrowheads indicate positions of primers developed in this study for specific PCR amplification of the desired region: $(\mathbf{C}$ and $\mathbf{D})$ mono specific primers and (E) double specific primers.
WGA574_36 chromosome 9 genomic contig; NW_ 925362.1 HsCraAADB02_461 chromosome 12 genomic contig (Chr 12-a); NW_925284.1 HsCraAADB02_454 chromosome 12 genomic contig (Chr 12-b); NT_ 011387.8 Hs20_11544 chromosome 20 genomic contig (Chr 20-a); NT_025215.4 Hs20_25371 chromosome 20 genomic contig (Chr 20-b); NW_001840455.1 HsUn_ WGA2630_36 genomic contig (CX); NW_001841138. 1 HsUn_WGA3313_36 genomic contig (Cy).

\section{Results}

\section{Case 1}

Sequence variations were identified for each PCR fragment that had an abnormal DHLPC pattern. The classical primers ${ }^{7}$ used in Poitiers Hospital allow amplification of exon 9 and the beginning of intron 9 (Figure 1, PCR product A). Sequencing of the PCR fragments using CF9.6 and CF9 + 58 primers revealed two heterozygous mutations, C. $1392+$ 6insC and C. $1392+12$ G $>$ A. However, PCR using the CF9-130 and CF9 + 58 primers (PCR product $B$, Bordeaux primers), revealed only the $G>T$ variation at position 1327, corresponding to the p.Asp443Tyr mutation, and not the expected c.1392 + 6insC and c.1392 + 12G $>$ A mutations.

To rule out the possibility that samples had been mishandled, the samples (and controls) were reassessed by PCR using the same primers and conditions; this reanalysis revealed the same mutations identified in the previous analysis. We then tested whether the choice of the primers could have affected the results. Surprisingly, only the p.Asp443Tyr mutation was detected using another primers pair that spans from intron 8 to intron 9, (CF9-268 and CF9 + 121, PCR C, from intron 8 to intron 9 as for Bordeaux primers).

To explain the results obtained, additional PCR amplifications followed by direct sequencing were performed using intronic or exonic primers (Table 1). The results revealed that PCR product F covers only the Asp443Tyr mutation, whereas PCR products obtained using exonic 
Table 2. Summary of Clones with Different Genotypes Used for PCR Amplification and Sequencing

\begin{tabular}{|c|c|c|c|c|c|c|}
\hline Case & $\begin{array}{l}\text { Clone } \\
\text { genotype }\end{array}$ & $\begin{array}{l}\mathrm{N} / \mathrm{N} \\
\mathrm{N} / \mathrm{N}\end{array}$ & $\begin{array}{c}\text { c.1327G }>\text { T(p.Asp443Tyr) } \\
\text { N/N }\end{array}$ & $\begin{array}{c}\mathrm{N} / \mathrm{N} \\
\text { c. } 1392+6 \mathrm{insC} \\
\text { c. } 1392+12 \mathrm{G}>\mathrm{A}\end{array}$ & $\begin{array}{c}\text { c. } 1327 \mathrm{G}>\mathrm{T}(\mathrm{p} . \text { Asp } 443 \mathrm{Tyr}) \\
\text { c.1392 + 6insC; } \\
\text { c. } 1392+12 \mathrm{G}>\mathrm{A}\end{array}$ & $\begin{array}{c}\text { Total } \\
\text { number of } \\
\text { clones } \\
\text { tested }\end{array}$ \\
\hline \multirow[t]{2}{*}{ Case 1} & $\%$ clones of PCR A & 19 & 54 & 27 & 0 & 26 \\
\hline & $\%$ clones of PCR C & 60 & 40 & 0 & 0 & 15 \\
\hline \multirow[t]{2}{*}{ Case 2} & $\%$ clones of PCR A & 4 & 26 & 35 & 35 & 15 \\
\hline & $\%$ clones of PCR C & 67 & 33 & 0 & 0 & 6 \\
\hline
\end{tabular}

$\mathrm{N} / \mathrm{N}=$ wild-type genotypes.

forward primers and intronic reverse primers cover the heterozygous c. $1392+$ 6insC and C.1392 + 12G>A mutations (data not shown).

To investigate the number of different alleles and confirm the unusual result from direct sequencing, PCR products of the region of interest have been cloned. Sequencing analysis of cloned $\mathrm{PCR}$ product $\mathrm{C}$ showed two alleles: one mutated ( $p$.Asp443Tyr allele) and one wild-type. In contrast, by using cloned PCR (A) product, a third allele is detected containing the c.1392 + 6insC and c.1392 + $12 \mathrm{G}>\mathrm{A}$ mutations. In addition, we found these apparent mutations in homozygous state, which clearly allows identification of the insertion. Table 2 shows the percentage of each genotype obtained by direct amplification and sequencing, confirming that different experimental protocol yielded o different numbers of alleles.

\section{Case 2}

Similar data have been obtained for case 2 either by direct sequencing or by subcloning of PCR products $A$ and $C$. This individual carries a $G>A$ polymorphism at position 1395 in exon 9. In fact, four alleles could be detected: two with $\mathrm{G}>\mathrm{A}$ at position 1395 and two with $\mathrm{G}>\mathrm{T}$ at position 1395 and C.1392 + 6insC; C.1392 + $12 \mathrm{G}>\mathrm{A}$. The c. $1392+$ GinsC and c. $1392+12 \mathrm{G}>\mathrm{A}$ mutations were never found without the $G>T$ polymorphism at position 1395.

Most surprisingly, these results show that a different sequence, an apparent copy of CFTR, was amplified specifically in each case. Indeed, the amplification procedure revealed a $\mathrm{G}>\mathrm{T}$ polymorphism at position 1395 in case 2 that was never observed in case 1 .

\section{DNA Sequence Comparisons}

A BLAST search for regions of similarity to CFTR exon 9 and its flanking introns revealed an identical sequence in five regions in the human genome, named 20-a, 20-b, 12-a, 12-b, 9, and two genomic contigs named $C x$ and Cy. An alignment of these sequences was preformed to determine the common region of similarity. The beginning of similarity is located $61 \mathrm{bp}$ upstream and continued 401 bp downstream of the exon 9 (Figure 1).

\section{Comparison of the CFTR Exon 9 Coding Sequence}

Exon 9 shares 95\% identity with other homologous sequences. Several variations have been identified in exon 9, including four possible mutations shown in Figure 2A: p.Lys464Asn, c.1328_1329delAT, c.1235delC, and p.Asn416ser (http://www.genet.sickkids.on.ca/cttr/; last accessed 24/12/2008) [Personal communications: p.Lys464Asn (E. Bleth, V. Gaston, P. Gautry), c.1328_1329delAT (T. Bienvenu, L. Tchertkoff, C. Cazeneuve, C Beldjord), c.1235delC (C. Férec), and p.Asn416Ser (L. Picci, M. Cameran, O. Marangon, D. Marzenta, M. Scarpa)]. Table 3 summarizes the mutations that may be due to these homologous sequences.

\section{Comparison of Intronic Sequences}

We also studied the relationship of the intronic boundaries of CFTR exon 9 with homologous sequences. The c. $1392+$ 6insC and c. $1392+12$ G $>$ A mutations, previously thought to lie at the beginning of intron 9 , were found in all homologous sequences (Figure 2B). Furthermore, an analysis of the number of thymines in a thymine repeat in intron 8 (IVS8) indicated that the duplicated sequences do not affect the T5/T7/T9 polythymidine tract situated in intron 8 near the acceptor splice site for exon 9. Moreover some other PCR products revealed addi-

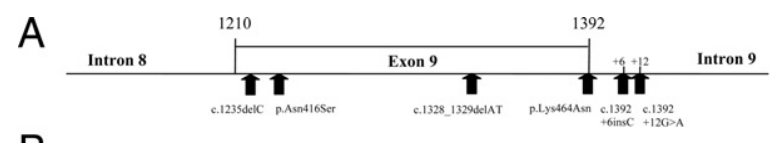

B

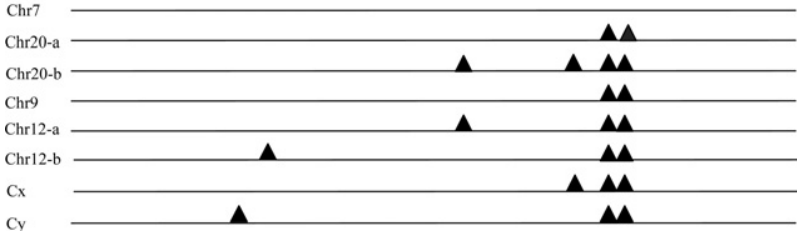

Figure 2. Mutations in human CFTR exon 9 , its intronic boundaries, and homologous sequences in the human genome. A: Name and localization of potentially incorrect mutations (pseudomutations). These variants could be a consequence of local sequence identity with similar regions in the human genome that result in a severely increased frequency of priming artifacts. B: Horizontal lines show human chromosomal regions with homology to CFTR exon 9 and its intronic boundaries, and arrowheads show positions of mutations found within these homologous sequences (based on CLUSTALW multiple alignment analysis). 
Table 3. Mutations in CFTR Exon 9 and its Intronic Boundaries that Have Homologous Sequences in Other Chromosomes

\begin{tabular}{|c|c|c|c|c|}
\hline CFTR mutation & $\begin{array}{c}\text { Common } \\
\text { nomenclature }\end{array}$ & Nucleotide change & Site of mutation* & Consequences \\
\hline p.Lys464Asn & $\mathrm{K} 464 \mathrm{~N}$ & G to $T$ at 1392 & Exon 9 (no. 10) & $\begin{array}{l}\text { Lys to Asn at } 464 ; \text { mRNA } \\
\text { splicing defect? }\end{array}$ \\
\hline c.1328_1329delAT & 1460delAT & Deletion of AT from 1328 & Exon 9 (no. 10) & Frameshift \\
\hline c. $1235 \mathrm{delC}$ & 1367delC & Deletion of $\mathrm{C}$ at 1235 & Exon 9 (no. 10) & Frameshift \\
\hline p.Asn416Ser & N416S & A to $G$ at 1247 & Exon 9 (no. 10) & Asn to Ser at 416 \\
\hline $\begin{array}{l}\text { c. } 1392+6 \text { insC; } \\
\text { c. } 1392+12 \mathrm{G}>\mathrm{A}\end{array}$ & $\begin{array}{l}1524+\text { 6ins } C \\
1524+12 G>A\end{array}$ & $\begin{array}{l}\text { Insertion of } C \text { after } 1392+ \\
6, G \text { to } A \text { at } 1392+12\end{array}$ & Intron 9 (no. 10) & mRNA splicing defect? \\
\hline
\end{tabular}

These mutations were previously reported as personal communication to the CF Genetic Analysis Consortium (http://www.genet.sickkids.on.ca/cftr, last accessed December 24, 2008).

${ }^{*}$ Conventional CFTR exon/intron numbering includes exons $6 \mathrm{a}$ and $6 \mathrm{~b}$, exons $14 \mathrm{a}$ and $14 \mathrm{~b}$, and exons $17 \mathrm{a}$ and 17b; for exon/intron numbers in parentheses, these exon pairs are numbered sequentially, without modifiers such as " $6 a$ " and " $6 \mathrm{~b} . "$

tional substitutions, indicating that other sequences were co-amplified (data not shown).

PCR analyses were performed using specific primers directed to the ends of or inside the homologous sequences to confirm the presence or absence of the C. $1392+$ 6insC and C. $1392+12 \mathrm{G}>$ A mutations (Figure 1). Sequencing of $P C R$ products $C, D$, and $E$ revealed the wild-type sequence of exon 9 on chromosome 7. Moreover, when the annealing temperature of PCR A was increased from $50^{\circ} \mathrm{C}$ to $60^{\circ} \mathrm{C}$, the PCR product A presented only the p.Asp443Tyr mutation ( $\mathrm{G}>\mathrm{T}$ at 1327$)$ in exon 9, and not the c.1392 + 6insC; c.1392 + 12G $>$ A mutations.

Furthermore, the c.1392 + 6insC and c.1392 + $12 \mathrm{G}>\mathrm{A}$ mutations were detected in DNA samples from each of the six negative control subjects and in patients with other CFTR mutations, when the classical primers were used, but were not detected in these same subjects when the specifically designed primers or the high annealing temperature was used. Taken together, our data confirm that the c.1392 + 6insC and c.1392 + 12G>A mutations are not actually present in the CFTR gene, but are in fact the consequence of multiple copies of this region throughout the human genome.

\section{Discussion}

The Cystic Fibrosis Mutation Database is useful for screening different populations, especially for laboratories involved in the diagnosis of CFTR mutations. Furthermore, to determine CFTR abnormalities in mutation carriers, these resources have important implications for continuing research on the molecular basis of CF. The identification and confirmation of newly discovered mutations is a challenge, particularly to CF clinics, for improving methods of detection.

The availability of complete genome sequences for many organisms in accessible databases has facilitated the assessment of DNA sequence similarities allowing a better understanding of both inter- and intragenomic homology, and thus of global genome structure. ${ }^{11}$ The final and conclusive characterization of a genomic rearrangement usually relies on the identification of sequences that harbor the rearrangement/mutation(s). ${ }^{12}$ Ultimately, the functional consequences of disease-associated genomic rearrangements/mutations can be assessed only by accurately determining the exact DNA sequences(s) responsible for the particular disease phenotype. BLAST analysis of CFTR exon 9 and its flanking intronic regions revealed several exon 9 repeat sequences in the human genome. Surprisingly, the c.1392 + 6insC and c.1392+ $12 \mathrm{G}>\mathrm{A}$ mutations were found in all of these pseudogene sequences. Moreover, the $\mathrm{T}$ at position 1395 was also detected in one similar sequence. Therefore, sequence similarities and structure alignment indicate that these mutations could be pseudomutations. It is noteworthy that we identified four other mutations in the CFTR database (http://www.genet.sickkids.on.ca/cftr/, last accessed December 24,2008 ) that are present in homologous sequences within CFTR exon 9. The above data are consistent with a report describing the amplification of this segment to multiple copies in human genome by cloning, sequence analysis, and chromosome localizations. ${ }^{5} \mathrm{~A}$ detailed analysis of this segment has previously shown that this region is part of a large duplicated sequence unit (LCR7-20), and that duplicated units are localized on different human chromosomes. Furthermore, restriction fragment analysis and limited sequencing data have shown that the human genome contains approximately 30 copies of LCR7-20-like sequences. ${ }^{6}$ Our present results, however, do not agree with this high copy number. Moreover, an insertion containing a 647-bp sequence with strong sequence similarity to CFTR exon 9 has been previously described, ${ }^{6}$ and the authors suggested that these regions were copied and co-integrated with an L1 retrotransposon during its transposition to new locations in the genome.

Based on our results and those from other studies, we suggest that technical anomalies can occur when screening for CFTR mutations due to the presence of several genomic regions that are homologous to this gene. Therefore, we have studied human CFTR exon 9 and its intronic boundaries by investigating a wide range of highly similar/homologous sequences to design primers that precisely amplify only CFTR exon 9 and its flanking introns, and that can be used to identify true mutations in this gene. We used an upstream fragment in intron 8 near exon 9 to avoid amplifying the polymorphic tract upstream of exon 9; other amplified fragments were located in exon 9 or intron 8 . This strategy successfully 
excluded amplification of pseudogene sequences. By direct sequencing with non-specific primers, the findings revealed different sequences that were not located in the CFTR exon 9 regions. Subsequently, we used PCR cloning with specific and non-specific primers to confirm the pseudogene variants. We have described here three specific primer pairs for CFTR exon 9 that have no similarity to other pseudogene sequences. In one primers set (CF-70, CF + 437), both the forward and reverse primers are outside the region of homology. In the two other primer sets, forward primers (CF-70, CF-268) that are outside the region of homology, and a common reverse primer $(\mathrm{CF}+121)$ is inside the region of homology. These designed primer sets, along with suitable melting temperatures, preclude co-amplification of pseudogene sequences.

Our results also suggest that several mutations that have been described in CFTR exon 9 and its flanking regions could, in fact, be ectopic variants in pseudogenes. These include p.Lys464Asn, c.1328_1329delAT, c.1235delC, and p.Asn416Ser in exon 9, and c.1392 + 6insC; c.1392 + 12G>A in intron 9. In conclusion, all patients carrying such mutations should be re-examined using the primers and protocol described here.

\section{References}

1. Tsui LC, Buchwald M: Cystic fibrosis. Ann Med Genet 1992, 24:192-245

2. Riordan JR, Rommens JM, Kerem BS, Alon N, Rozmahel R, Grzelczak Z, Zielenski J, Lok S, Plavsic N, Chou JL, Drumm ML, Iannuzzi MC,
Collins FS, Tsui LC: Identification of the cystic fibrosis gene: cloning and characterization of complementary DNA. Science 1989 , 245:1066-1073

3. Wang LJ, Wang J, Zhang YH, Hsu E, Heim RA, Bowman CM, Woo MS: Improved detection of CFTR mutations in Southern California Hispanic CF patient. Hum Mutat 2001, 18:296-307

4. Bobadilla JL, Macek JM, Fine JP, Farrell PM: Cystic fibrosis: a worldwide analysis of CFTR mutation-correlation with incidence data and application to screening. Hum Mutat 2002, 19:575-606

5. Rozmahel R, Heng HHO, Duncan AMV, Shi XM, Rommens JM, Tsui LC: Amplification of CFTR exon 9 sequences to multiple locations in the human genome. Genomics 1997, 45:554-561

6. Liu X, Li X, Li M, Acimovic YJ, Li Z, Tshui LC: Characterization of the segmental duplication LCR7-20 in the human genome. Genomics 2004, 83:262-269

7. Le Marechal C, Audrezet MP, Quere I, Raguenes O, Langonne S, Ferec $\mathrm{C}$ : Complete and rapid scanning of the cystic fibrosis transmembrane conductance regulator (CFTR) gene by denaturing highperformance liquid chromatography (D-HPLC): major implications for genetic counseling. Hum Genet 2001, 108:290-288

8. Strong TV, Wilkinson DJ, Monsoura MK, Devor DC, Henze K, Yang Y, Wilson JM, Cohn JA, Dawson DC, Frizzell RA, Collins FS: Expression of an abundant alternatively spliced form of the cystic fibrosis transmembrane conductance regulator (CFTR) gene is not associated with a cAMP-activated chloride conductance. Hum Mol Genet 1993, 2:225-230

9. Altschul SF, Gish W, Miller W, Myers EW, Lipman DJ: Basic local alignment search tool. J Mol Biol 1990, 215:403-410

10. Altschul SF, Madden TL, Schaffer AA, Zhang J, Zhang Z, MillerW, Lipman DJ Gapped: BLAST and PSI-BLAST: a new generation of protein database search programs. Nucleic Acids Res 1997, 25:3389-3402

11. Miller W: Comparison of genomic DNA sequences: solved and unsolved problems. Bioinformatics 2001, 17:391-397

12. Montagna R: Genomic rearrangement account for more than onethird of the BRCA1 mutations in the northern Italian breast/ ovarian cancer families. Hum Mol Genet 2003, 12:1055-1061 